\title{
Effect of Transversus Abdominis Plane Block on Postoperative Pain after Colorectal Surgery: A Meta-Analysis of Randomized Controlled Trials
}

\author{
Lin Liu Yan-Hu Xie Wei Zhang Xiao-Qing Chai \\ Department of Anesthesiology, An Hui Provincial Hospital affiliated to An Hui Medical University, He Fei, China
}

\section{Significance of the Study}

- The effect of transversus abdominis plane (TAP) block on postoperative pain remains uncertain. This meta-analysis aimed to assess the analgesic efficacy of TAP block in patients undergoing colorectal surgery (CRS). Based on 7 clinical trials, we found that TAP block reduced postoperative acute pain and morphine consumption. This study provides evidence for the application of TAP block in patients who undergo CRS.

\section{Keywords}

Transversus abdominis plane block · Colorectal surgery ·

Postoperative pain · Analgesic efficacy

\begin{abstract}
Objectives: To assess the analgesic efficacy of transversus abdominis plane (TAP) block in patients undergoing colorectal surgery (CRS). Materials and Methods: The databases of PubMed, ISI Web of Science, and Embase were searched, and randomized controlled studies (RCTs) that compared TAP block to control for relief of postoperative pain in patients who underwent CRS were included. Outcomes, including postoperative pain at rest and with movement, morphine use, postoperative nausea and vomiting, and the length of hospital stay, were analyzed using STATA software. The weighted mean differences (WMDs) with $95 \%$ confidence intervals $(95 \% \mathrm{Cls})$ or relative risk with $95 \% \mathrm{Cl}$ were used to present the strength of associations. Results: A total of 7 RCTs with 511 patients were included. The results of this study suggested that TAP block significantly relieved post-
\end{abstract}

\begin{tabular}{ll}
\hline KARGER & $\begin{array}{l}\text { ( 2018 The Author(s) Karger } \\
\text { Published by S. Karger AG, Basel }\end{array}$ \\
E-Mail karger@karger.com & $\begin{array}{l}\text { This is an Open Access article licensed under the Creative Commons } \\
\text { Attribution-NonCommercial-4.0 International License (CC BY-NC) } \\
\text { (http://www.karger.com/Services/OpenAccessLicense), applicable to } \\
\text { the online version of the article only. Usage and distribution for com- } \\
\text { mercial purposes requires written permission. }\end{array}$
\end{tabular}

operative pain during postanesthetic recovery after CRS at rest and during movement (WMDs were $-0.98[95 \% \mathrm{Cl}-1.57$ to -0.38 ] and -0.68 [ -1.07 to -0.30$]$, respectively), and also decreased pain intensity during movement $24 \mathrm{~h}$ after CRS (WMD: -0.57 [95\% Cl -1.06 to -0.08$]$ ). TAP block significantly reduced opioid consumption within $24 \mathrm{~h}$ when compared to controls, with a WMD of $15.66(95 \% \mathrm{Cl}-23.93$ to -7.39$)$. However, TAP block did not shorten the length of hospital stay. Conclusions: TAP block was an effective approach for relief of postoperative pain and reduced postoperative consumption of morphine. More RCTs with large sample sizes are required to confirm these findings. 02018 The Author(s)

Published by S. Karger AG, Basel

\section{Introduction}

Postoperative pain is of great concern to patients who undergo colorectal surgery (CRS), and acute pain relief after surgery is critical to facilitate the recovery of bowel function and to shorten the length of hospital 
stay $[1,2]$. Presently, opioids are the major drugs used for postoperative pain relief [3], but they lead to significant adverse effects, including nausea, vomiting, urinary retention, and delayed recovery of bowel mobility [4].

Transversus abdominis plane (TAP) block involves infiltration of local anesthetics into the fascial plane between the internal oblique and transversus abdominis muscle, and it has been suggested to be an effective approach for relieving postoperative pain after lower abdominal surgery with minimal adverse effects $[5,6]$. A meta-analysis of randomized controlled trials (RCTs) for a variety of laparoscopic surgeries indicated that TAP block reduced postoperative pain and drug consumption [7]. More recently, a meta-analysis that included 7 randomized controlled trials for laparoscopic cholecystectomy suggested that ultrasound-guided TAP block reduced the intensity of postoperative consumption of opioids and decreased the incidence of nausea and vomiting after surgery [8].

Several clinical trials have reported the effect of TAP block in patients who underwent CRS [2, 9-14]; however, the results of these studies were conflicting. A clinical study conducted by Tikuisis et al. [2] on 64 patients undergoing elective colonic resection for cancer reported that TAP block was effective in reducing postoperative pain and shortening the length of hospital stay. Another clinical trial by Torup et al. [13] found that TAP block did not relieve postoperative pain but reduced opioid use after laparoscopic hemicolectomy. However, 2 clinical trials suggested that TAP block appeared to be a safe intervention but provided no specific advantage in CRS [12, 14]. This meta-analysis was conducted in order to determine whether TAP block is an effective approach for the relief of postoperative pain.

\section{Materials and Methods}

This meta-analysis was conducted according to the preferred reporting items for systematic reviews and meta-analyses statement (PRISMA-2009) [15].

\section{Literature Search and Study Selection}

Two authors (Lin Liu and Yan-Hu Xie) searched the electronic databases of PubMed, ISI Web of Science, and Embase up to February 1,2017 . The following key words were used for the search: (transversus abdominis plane block) AND (colon OR colorectal OR colectomy OR rectal) AND ((randomized controlled trial (RCT)) OR (clinical trial)). Reference lists of the included studies and relevant reviews that were published in the past 5 years were screened to identify additional publications.

TAP Block and Postoperative Anesthesia

\section{Inclusion and Exclusion}

The inclusion criteria were as follows: (1) study compared TAP block to an inactive control like placebo or no intervention in patients who underwent CRS for benign or malignant colorectal diseases; (2) both open and laparoscopy CRSs were included in this study; (3) outcomes included postoperative pain intensity, opioid consumption, occurrence of adverse events, and length of hospital stay; (4) the study must be an RCT and published in English. The exclusion criteria were: (1) comparison of TAP block with wound infiltration, and (2) unavailability of full text.

\section{Data Extraction and Collection}

Two authors (Lin Liu and Wei Zhang) extracted the data from the included studies using a predefined form. The following data were extracted: the name of the first author, publication year, country of patients, sample size, types of colorectal diseases, intraoperative anesthesia, postoperative analgesics, postoperative pain intensity at rest and with movement, postoperative opioid use, length of hospital stay, and the incidences of nausea and vomiting. Disagreements relating to data extraction were resolved by discussion with the third author (Yan-Hu Xie).

\section{Primary and Secondary Outcomes}

The primary outcome was postoperative pain intensity at rest and with movement. The pain intensity was assessed using a visual analog scale or a verbal rating scale from 0 to 10 . The secondary outcomes were postoperative opioid use, number of patients with postoperative nausea and vomiting (PONV), and length of hospital stay. Opioid consumption was transformed to morphineequivalent consumption according to previously published studies (morphine $1 \mathrm{mg}=$ tramadol $10 \mathrm{mg}$ and morphine $10 \mathrm{mg}=$ fentanyl $1 \mathrm{mg}$ i.v.), if possible [8].

\section{Risk of Bias Assessment}

Two authors (Liu Liu and Wei Zhang) assessed the risk of bias independently using the Cochrane Collaboration Risk of Bias tool. This tool contains 6 different domains, including "random sequence generation," "allocation concealment," "blinding of participants and personnel," "blinding of outcome assessment," "incomplete outcome data," and "selective reporting." The estimated risk of bias for each included trial was categorized as "low," "unclear," or "high." Disagreements on bias assessment were resolved by discussion with the third author (Yan-Hu Xie).

\section{Statistical Analysis}

Continuous outcomes were reported as weighted mean differences (WMDs) and 95\% confidence intervals (95\% CIs), while categorical outcomes were reported as risk ratios and 95\% CIs; $p<$ 0.05 indicated statistical significance. If the data were presented as values other than the mean with standard deviation, they were transformed into the mean and standard deviation according to previously reported methodology $[16,17]$ to allow more studies for combined analysis. Between-study heterogeneity was assessed using the Q test and $I^{2}$ test, and $p<0.10$ or $I^{2}>50 \%$ suggested the existence of between-study heterogeneity; a random-effect model was used if heterogeneity existed; otherwise, a fixed-effect model was applied [18]. In addition, the source of between-study heterogeneity was assessed using a Galbraith plot. Publication bias was assessed using the Egger test, and $p<0.05$ indicated publication bias among the included studies. In order to improve the robust- 
ness of results, combined analyses, sensitivity analysis, and test for publication bias were conducted if 4 or more studies were included for an outcome.

All statistical analyses were conducted using STATA 10.0 (StataCorp, College Station, TX, USA). All statistical tests were 2-sided.

\section{Results}

\section{Description of Included Trials}

A total of 18 studies were identified; by screening titles, abstracts and full texts, 7 clinical trials with 511 patients (251 patients in the TAP block group and 260 patients in the control group) were included (online suppl. Fig. 1; see www.karger.com/doi/10.1159/000487323 for all online suppl. material) [2, 9-14]. The characteristics of the included studies are shown in online supplementary Table 1. Sample sizes ranged from 40 to 147 patients. Among the included studies, 5 reported that TAP block was performed after the induction of anesthesia [2, 10, 12-14], and 2 studies reported that TAP block was conducted at the end of surgery $[9,11]$. In addition, ultrasound-guided TAP block was conducted in 5 trials, and conventional TAP block was performed in $2[9,11]$. The risks of bias for each included study are assessed, and results are shown in online supplementary Table 2. The qualities of all included studies were high, with a low risk of bias.

\section{Postoperative Pain Intensity}

Postoperative pain intensity was analyzed during the period of postanesthetic recovery (PAR) that was defined as $2 \mathrm{~h}$ after surgery and $24 \mathrm{~h}$ after surgery at rest and during movement. A total of $5[2,10,11,13,14]$ and 4 trials $[2,10,13,14]$ were included for analysis of pain intensity during PAR at rest and during movement, respectively. Pain intensity was significantly decreased in patients receiving TAP block as compared to controls during PAR after surgery at rest and during movement $\left(\mathrm{WMD}_{\text {rest }}\right.$ : -1.10 [95\% CI -1.43 to -0.76 ], $p<0.001$; pheterogeneity $=$ 0.082 and $I^{2}=51.6 \%$; WMD movement -0.68 [95\%CI -1.07 to -0.30$], p<0.001 ;$ pheterogeneity $=0.777$ and $I^{2}=0 \%$ ) (Fig. 1a, b). In addition, TAP block also significantly decreased pain intensity $24 \mathrm{~h}$ after surgery during movement ( $\mathrm{WMD}_{\text {movement }}$-0.57 [95\% CI -1.06 to -0.08 ], $p=$ 0.022 ; peterogeneity $=0.278$ and $I^{2}=22.2 \%$ ) (Fig. 1c); however, there was no statistical significance $24 \mathrm{~h}$ after surgery at rest $\left(\mathrm{WMD}_{\text {rest }}:-0.33\right.$ [95\% CI -0.70 to 0.05$], p=$ 0.087; pheterogeneity $=0.455$ and $\left.I^{2}=0 \%\right)$ (Fig. 1d).

\section{Postoperative Opioid Use}

Six studies [2, 9, 10, 12-14] reported opioid use after CRS. Combined analysis suggested that TAP block significantly reduced opioid use after CRS (WMD: -15.66 [95\% CI -23.93 to -7.39$], p<0.001 ; p_{\text {heterogeneity }}=0.001$ and $I^{2}=75.2 \%$ ) (Fig. 2). Between-study heterogeneity was observed using the Galbraith plot; the study of Tikuisis et al. [2] was the major source of between-study heterogeneity. After excluding this study, the degree of heterogeneity decreased ( $p_{\text {heterogeneity }}=0.158$ and $I^{2}=39.5 \%$ ), and the result of reanalysis suggested that TAP block contributed to reduce the use of opioids as compared to patients in the control group (WMD: -10.55 [95\% CI -13.09 to -8.01 , $p<0.001)$.

\section{Postoperative Nausea and Vomiting}

A total of 4 trials $[2,9,12,13]$ were available for analysis of PONV. Combined analysis suggested that patients in the TAP block group had a comparable risk of PONV with patients in the control group (risk ratio: 1.02 [95\% CI 0.81-1.03], $p=0.842 ; p_{\text {heterogeneity }}=0.624$ and $I^{2}=0 \%$ ) (Fig. 3).

\section{Length of Hospital Stay}

Four studies $[2,10,11,14]$ reported the impact of TAP block on the length of hospital stay and were included for combined analysis. The length of hospital stay was not statistically different between patients in the TAP block group and in the control group (WMD: -0.02 [95\% CI -1.81 to 1.78 ], $p=0.821$; pheterogeneity $=0.002$ and $I^{2}=$ $79.8 \%$ ) (Fig. 4). Between-study heterogeneity was observed using the Galbraith plot; the study of Tikuisis et al. [2] was the major source of between-study heterogeneity for postoperative opioid use. Recombined analysis was not conducted as only 3 studies remained after excluding this study.

\section{Safety of TAP Block Procedure}

Of the 7 clinical trials included, 3 studies reported information regarding the safety of the TAP block procedure. All 3 studies reported that TAP block was easily performed and safe; no adverse event was reported re-

Fig. 1. Effect of transversus abdominis plane block on postoperative pain during postanesthetic recovery (PAR) and $24 \mathrm{~h}$ after colorectal surgery. a Postoperative pain during PAR after surgery at rest. b Postoperative pain during PAR after surgery during movement. c Postoperative pain $24 \mathrm{~h}$ after surgery at rest. d Postoperative pain $24 \mathrm{~h}$ after surgery during movement.

(For figure see next page.)
Liu/Xie/Zhang/Chai 


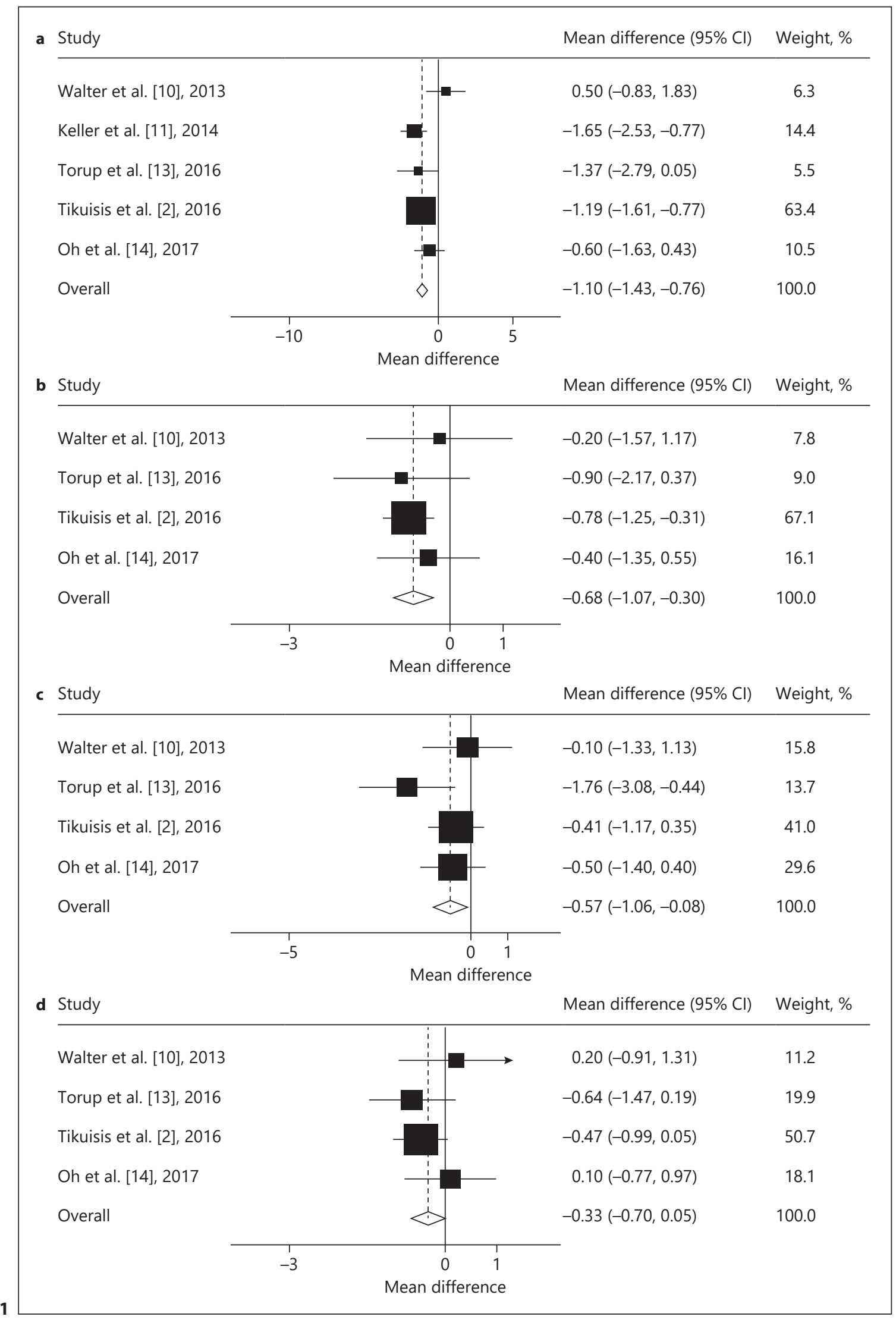




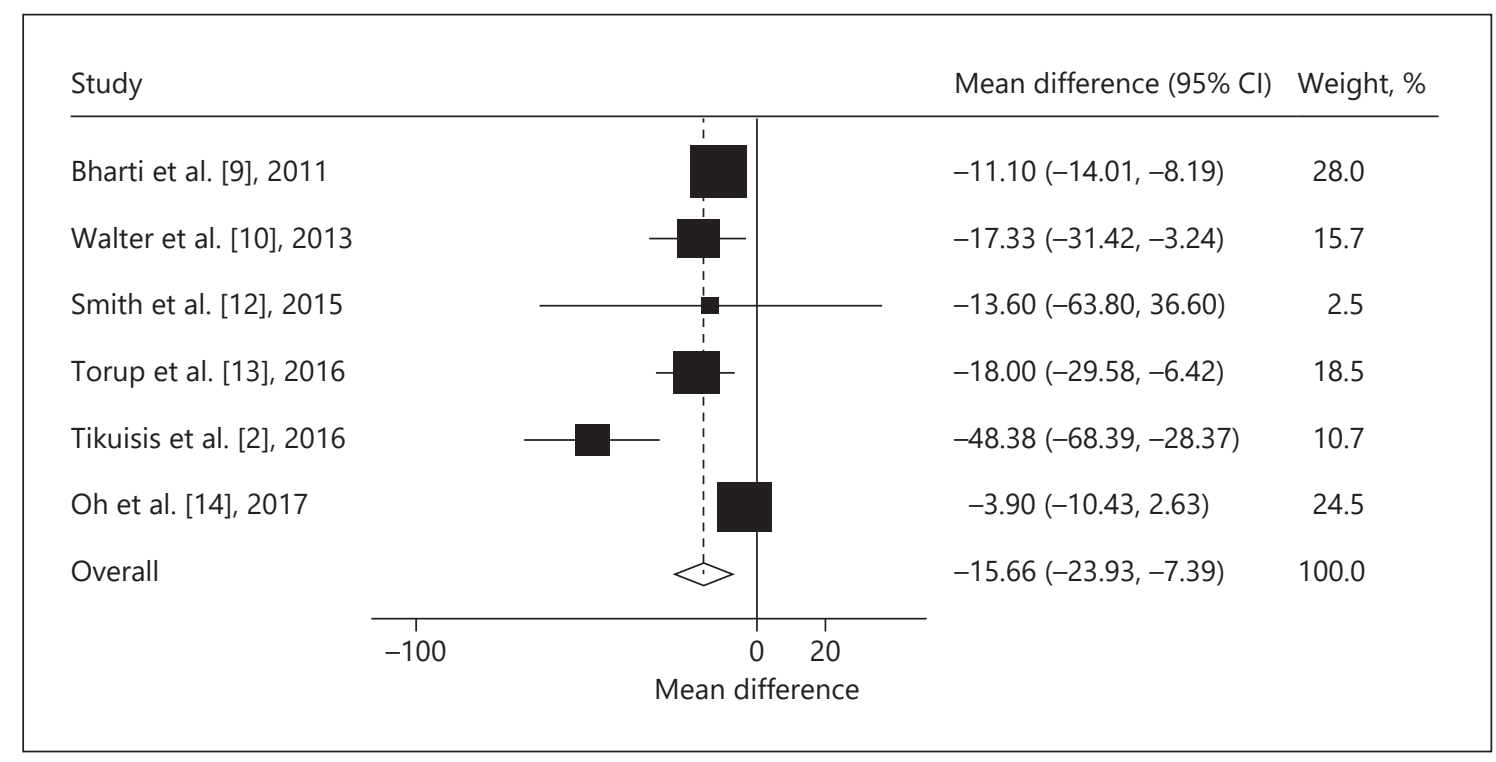

Fig. 2. The impact of transversus abdominis plane block on opioid use after colorectal surgery.

lated to the TAP block procedure or anesthetic-related toxicity.

\section{Assessment of Publication Bias}

By the Egger test, publication bias was not observed for the combined analyses ( $p=0.718$ and 0.402 for pain intensity $2 \mathrm{~h}$ after surgery at rest and with movement, respectively; $p=0.342$ and 0.498 for pain intensity $24 \mathrm{~h}$ after surgery at rest and with movement, respectively; $p=$ $0.329,0.236$ and 0.812 for postoperative morphine use, PONV, and length of hospital stay, respectively).

\section{Discussion}

TAP block has been performed in conventional open and laparoscopic CRS in several clinical trials [2, 9-14]; however, its effect has remained controversial. Therefore, we conducted this meta-analysis based on 7 clinical trials with 511 patients. The primary outcome was postoperative pain intensity during PAR and $24 \mathrm{~h}$ after surgery at rest and with movement. We also analyzed the postoperative opioid consumption, incidence of PONV, and length of hospital stay. The results of this study suggested that TAP block significantly relieved pain intensity during PAR and $24 \mathrm{~h}$ after CRS, when compared to patients who received placebo or were without TAP block. In addition, TAP block also decreased opioid use. However,
TAP block neither reduced the incidence of PONV nor did it shorten the length of hospital stay. In addition, no adverse event associated with TAP block or anestheticrelated toxicity was reported. These observations suggest that TAP block provides analgesia in the early postoperative period and a morphine-sparing effect, which may contribute to the goals of enhanced recovery after surgery pathways in CRS. Besides, TAP blocks are easily performed and cost-effective, with minimal procedure-related morbidity.

TAP block has been proven to be effective for the reduction of postoperative pain intensity after lower abdominal surgery under the umbilicus, especially within 24 $h$ after surgery [19-21]. This meta-analysis suggested that TAP block significantly reduced pain intensity during PAR after CRS both at rest and during movement; however, it only reduced pain $24 \mathrm{~h}$ after surgery during movement but not at rest. Similar pain relief has been reported for other abdominal surgeries $[19,22]$. A clinical trial conducted by Røjskjaer et al. [22] suggested that TAP block significantly reduced pain intensity $8 \mathrm{~h}$ after abdominal hysterectomy. Peng et al. [8] conducted a meta-analysis on the impact of TAP block on postoperative analgesia after laparoscopic cholecystectomy; the results of this study suggested that TAP block is effective for relief of postoperative pain and reducing opioid consumption [8].

The effect of TAP block on postoperative late pain measured $24 \mathrm{~h}$ after CRS has been controversial. Consid- 


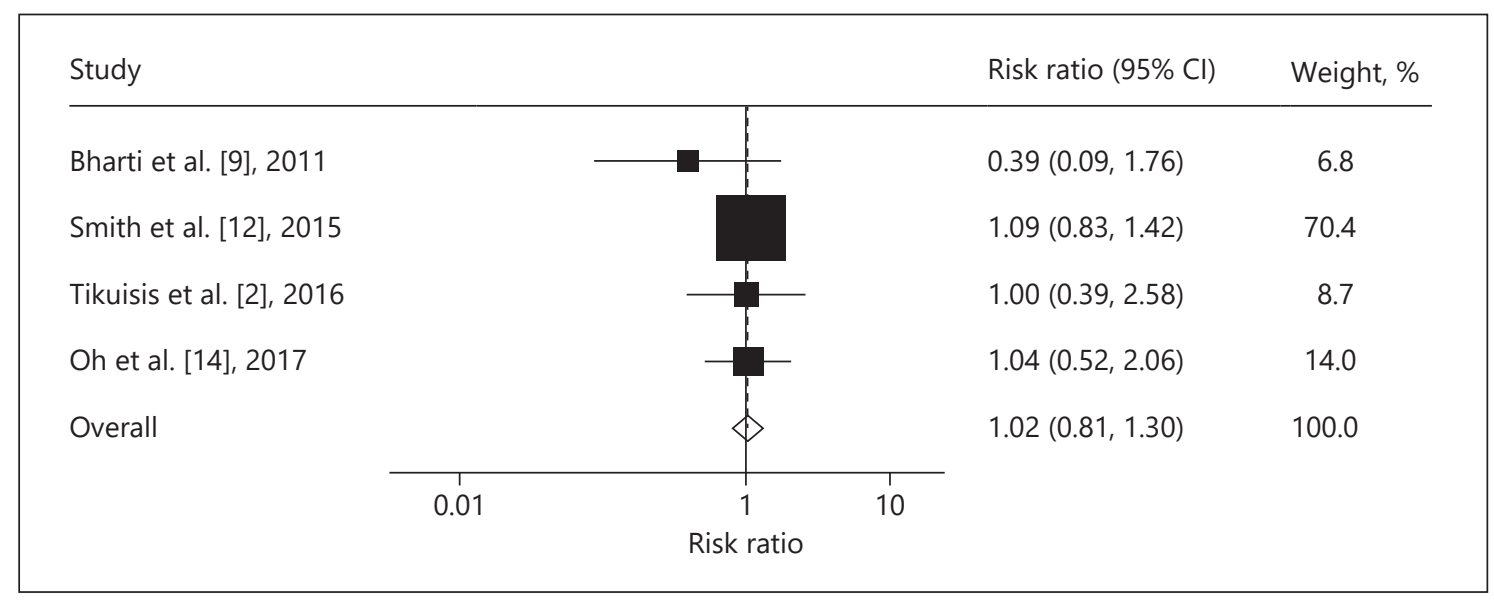

Fig. 3. Incidence of postoperative nausea and vomiting in patients and controls.

\begin{tabular}{|c|c|c|c|c|}
\hline Study & & & Mean difference $(95 \% \mathrm{CI})$ & Weight, \% \\
\hline Walter et al. [10], 2013 & & & $1.08(-0.58,2.74)$ & 28.6 \\
\hline Keller et al. [11], 2014 & & & $0.75(0.03,1.47)$ & 35.7 \\
\hline Tikuisis et al. [2], 2016 & & & $-2.16(-3.53,-0.79)$ & 31.0 \\
\hline Oh et al. [14], 2017 & & - & $1.66(-6.17,9.49)$ & 4.6 \\
\hline \multirow[t]{2}{*}{ Overall } & & & $-0.02(-1.81,1.78)$ & 100.0 \\
\hline & -10 & $\begin{array}{c}01 \\
\text { Mean difference }\end{array}$ & & \\
\hline
\end{tabular}

Fig. 4. Length of hospital stay of patients and controls.

ering the duration of conventional local anesthetics like bupivacaine and ropivacaine used in TAP block, it appeared to be ineffective in late pain. However, a published meta-analysis [7] as well as our study suggested that TAP block reduced late pain during movement. This could be explained by 2 reasons. First, preoperative TAP block reduced the central sensitization of pain caused by surgical stimulation, which is also known as "preemptive analgesic effect" [23]. Secondly, the duration of local anesthetics was possibly different when they were in blood or used for nerve blocking [24]. Therefore, the kind and optimal dose of the local anesthetic used in TAP block should be further studied.

TAP Block and Postoperative Anesthesia
Although most published trials reported that TAP block was effective for pain relief after CRS, a few studies suggested that TAP block did not offer enough advantage for clinical use $[12,14]$. Several explanations have been put forward for the ineffectiveness of TAP block in CRS. First, according to the results of a magnetic resonance imaging study and a cadaver study [19-21], TAP block produced a block of sensory dermatomes from $T_{10}$ to $L_{1}$, which represented a region of the lower abdomen under the umbilicus; however, it did not cover the region of the upper abdomen. Two studies reported that an incision was produced in the upper abdomen for right colectomy, and TAP block did not work effectively in patients undergoing right colectomy $[12,14]$. Second, pain after CRS 
included parietal pain, induced by incision of the abdominal wall, and visceral pain. It has been recognized that TAP block could relieve parietal pain; however, it had no effect on visceral pain, which might decrease the effect of TAP block [14].

Anesthetic drugs like opioids have been widely used for pain relief after surgery, but they induce many adverse effects, like nausea, vomiting, ileus, and immobilization. Therefore, reducing opioid use is beneficial for earlier recovery of bowel function and higher satisfaction. The present study suggested that TAP block significantly reduced opioid consumption after CRS, especially on the first day after surgery. This finding is consistent with some clinical trials, which reported that TAP block reduced opioid consumption in patients undergoing other lower abdominal surgeries $[6,25]$.

Most published clinical trials have focused on the impact of TAP block on postoperative pain relief and opioid consumption; however, whether TAP block contributed to the recovery of gastrointestinal function and the reduced length of hospital stay after CRS remained unclear. Of the 7 studies included, 2 reported the effect of TAP block on the recovery of gastrointestinal function $[2,12]$, and 4 trials reported the length of hospital stay $[2,10,11$, 14]. A study conducted by Smith et al. [12] suggested that TAP block did not affect the recovery of gastrointestinal function and length of hospital stay, including postoperative flatus and bowel movement. In contrast, Tikuisis et al. [2] reported that TAP block significantly enhanced bowel movement and shortened the length of hospital study [14]. Our study concluded that TAP block did not have a significant impact on the length of hospital stay. Because only 2 trials assessed the impact of TAP block on the recovery of gastrointestinal function, combined analysis could not be conducted, and more clinical trials are needed to substantiate this.

A recent meta-analysis to assess the impact of TAP block on laparoscopic CRS showed that TAP block re- duced postoperative pain during PAR with movement and did not reduce postoperative pain $24 \mathrm{~h}$ after CRS and opioid consumption [26]. There were several reasons for the difference in results between the study of Oh et al. [26] and ours. First, our study focused on the impact of TAP block on postoperative pain after CRS (both open and laparoscopic surgery). Second, this meta-analysis included RCT studies only. In contrast, the study by Oh et al. included both retrospective and RCT studies, which reduced the robustness of results. Third, we included an additional study reported by Torup et al. [13], which also met the inclusion criteria of our study.

There were some limitations to the current study. First, the sample size was relatively small, which might decrease the robustness of the results. However, this study was analyzed on the basis of 7 RCTs with a low risk of bias, which enhanced the reliability of the results. Second, TAP block was suggested to be more effective for lower abdominal surgery with incision under the umbilicus than upper abdominal surgery. Right-sided and left-sided colectomy required incision in the upper abdomen, while sigmoid resection and rectal surgery needed incisions in the lower abdomen. It appears that TAP block may be more effective for sigmoid and rectal surgery than colonic surgery; therefore, it is more reasonable to assess the impact of TAP block on colonic surgery and rectal surgery separately. Third, a few studies assessed the impact of TAP block on the recovery of gastrointestinal function after CRS, and no clear conclusions were made. Therefore, more clinical trials are needed.

In summary, based on the 7 clinical trials, this study suggested that TAP block was effective in reducing the intensity of pain and consumption of opioids after CRS. However, TAP block did not shorten the length of hospital stay. TAP block seems to be safe for clinical application as no adverse events including anesthetic-related toxicity were reported.

\section{References}

1 Buvanendran A, Kroin JS: Multimodal analgesia for controlling acute postoperative pain. Curr Opin Anaesthesiol 2009;22:588-593.

2 Tikuisis R, Miliauskas P, Lukoseviciene V, et al: Transversus abdominis plane block for postoperative pain relief after hand-assisted laparoscopic colon surgery: a randomized, placebo-controlled clinical trial. Tech Coloproctol 2016;20:835-844.
3 Cheng YJ: Lidocaine SKIN PAtch (Lidopat ${ }^{\circledR}$ $5 \%)$ is effective in the treatment of traumatic rib fractures: a prospective double-blinded and vehicle-controlled study. Med Princ Pract 2016;25:36-39.

4 Kehlet H, Rung GW, Callesen T: Postoperative opioid analgesia: time for a reconsideration? J Clin Anesth 1996;8:441-445.
5 Rafi AN: Abdominal field block: a new approach via the lumbar triangle. Anaesthesia 2001;56:1024-1026.

6 Abdallah FW, Laffey JG, Halpern SH, et al: Duration of analgesic effectiveness after the posterior and lateral transversus abdominis plane block techniques for transverse lower abdominal incisions: a meta-analysis. Br J Anaesth 2013;111:721-735. 
7 De Oliveira GS Jr, Castro-Alves LJ, Nader A, et al: Transversus abdominis plane block to ameliorate postoperative pain outcomes after laparoscopic surgery: a meta-analysis of randomized controlled trials. Anesth Analg 2014; 118:454-463.

8 Peng K, Ji FH, Liu HY, et al: Ultrasound-guided transversus abdominis plane block for analgesia in laparoscopic cholecystectomy: a systematic review and meta-analysis. Med Princ Pract 2016;25:237-246.

9 Bharti N, Kumar P, Bala I, et al: The efficacy of a novel approach to transversus abdominis plane block for postoperative analgesia after colorectal surgery. Anesth Analg 2011;112: 1504-1508.

10 Walter CJ, Maxwell-Armstrong C, Pinkney $\mathrm{TD}$, et al: A randomised controlled trial of the efficacy of ultrasound-guided transversus abdominis plane (TAP) block in laparoscopic colorectal surgery. Surg Endosc 2013;27: 2366-2372.

11 Keller DS, Ermlich BO, Schiltz N, et al: The effect of transversus abdominis plane blocks on postoperative pain in laparoscopic colorectal surgery: a prospective, randomized, double-blind trial. Dis Colon Rectum 2014;57: 1290-1297.

12 Smith SR, Draganic B, Pockney P, et al: Transversus abdominis plane blockade in laparoscopic colorectal surgery: a double-blind randomized clinical trial. Int J Colorectal Dis 2015;30:1237-1245.

13 Torup H, Hansen EG, Bogeskov M, et al: Transversus abdominis plane block after laparoscopic colonic resection in cancer patients: a randomised clinical trial. Eur J Anaesthesiol 2016;33:725-730
14 Oh TK, Yim J, Kim J, et al: Effects of preoperative ultrasound-guided transversus abdominis plane block on pain after laparoscopic surgery for colorectal cancer: a doubleblind randomized controlled trial. Surg Endosc 2017;31:127-134.

15 Liberati A, Altman DG, Tetzlaff J, et al: The PRISMA statement for reporting systematic reviews and meta-analyses of studies that evaluate health care interventions: explanation and elaboration. Ann Intern Med 2009; 151:W65-W94.

16 Hozo SP, Djulbegovic B, Hozo I: Estimating the mean and variance from the median, range, and the size of a sample. BMC Med Res Methodol 2005;5:13.

17 Wan X, Wang W, Liu J, et al: Estimating the sample mean and standard deviation from the sample size, median, range and/or interquartile range. BMC Med Res Methodol 2014;14:135.

18 DerSimonian R, Laird N: Meta-analysis in clinical trials. Control Clin Trials 1986;7:177-188.

19 Abu Elyazed MM, Mostafa SF, Abdullah MA, et al: The effect of ultrasound-guided transversus abdominis plane (TAP) block on postoperative analgesia and neuroendocrine stress response in pediatric patients undergoing elective open inguinal hernia repair. Paediatr Anaesth 2016;26:1165-1171.

20 Borglum J, Jensen K, Christensen AF, et al: Distribution patterns, dermatomal anesthesia, and ropivacaine serum concentrations after bilateral dual transversus abdominis plane block. Reg Anesth Pain Med 2012;37:294-301.
21 Tran TM, Ivanusic JJ, Hebbard P, et al: Determination of spread of injectate after ultrasound-guided transversus abdominis plane block: a cadaveric study. Br J Anaesth 2009; 102:123-127.

22 Røjskjaer JO, Gade E, Kiel LB, et al: Analgesic effect of ultrasound-guided transversus abdominis plane block after total abdominal hysterectomy: a randomized, double-blind, placebo-controlled trial. Acta Obstet Gynecol Scand 2015;94:274-278.

23 Munteanu AM, Cionac Florescu S, Anastase $\mathrm{DM}$, et al: Is there any analgesic benefit from preoperative versus postoperative administration of etoricoxib in total knee arthroplasty under spinal anaesthesia? A randomised double-blind placebo-controlled trial. Eur J Anaesthesiol 2016;33:840-845.

24 Calder K, Chung B, O’Brien C, et al: Bupivacaine digital blocks: how long is the pain relief and temperature elevation? Plast Reconstr Surg 2013;131:1098-1104.

25 Kanazi GE, Aouad MT, Abdallah FW, et al: The analgesic efficacy of subarachnoid morphine in comparison with ultrasound-guided transversus abdominis plane block after cesarean delivery: a randomized controlled trial. Anesth Analg 2010;111:475-481.

26 Oh TK, Lee SJ, Do SH, et al: Transversus abdominis plane block using a short-acting local anesthetic for postoperative pain after laparoscopic colorectal surgery: a systematic review and meta-analysis. Surg Endosc 2018;32:545552 